\title{
Que reste-t-il du textile vosgien ?
}

\section{Textile in the Vosges mountain: does is still exist?}

\author{
Simon EDELBLUTTE \\ Université Nancy \\ Département de Géographie \\ CERPA - Centre de Recherches et d'Études sur les Paysages \\ BP 33-97 \\ 54015 NANCY Cedex \\ 0383968421 / 0682245699 \\ simon.edelblutte@univ-nancy2.fr
}

\section{Résumé :}

Dans un contexte peu favorable de concurrence internationale exacerbée, le secteur textile semble aujourd'hui en voie de disparition dans le Massif vosgien, dont il est pourtant une activité emblématique depuis le XIXème siècle. Cependant, si ce déclin est réel et largement perceptible économiquement, socialement et spatialement, le textile reste l'un des piliers industriels du massif. Ce travail, après avoir évoqué le poids toujours actuel du textile vosgien, puis ses difficultés, insiste sur les solutions mises en œuvre pour préserver et développer les sites encore en fonction, avant enfin d'évoquer les bouleversements spatiaux liés à cette reconversion de longue haleine.

\section{Mots-clés :}

Industrie, textile, mondialisation, reconversion, Massif vosgien, Lorraine, Alsace, France

\section{Abstract :}

Within the frame of globalisation, textile branch seems to be near of total disappearance in the Vosges mountain, although this industry was the foundation of the region's industrialisation during $19^{\text {th }}$ century and early $20^{\text {th }}$ century. However, if the decline of textile industry is real both economically and spatially, it's staying a very important activity in the Vosges mountain. Firstly, this work aims to show the weight and the difficulties of this industrial branch before to mention the solutions to preserve and develop the remains of this activity. Finally, it deals with spatial reorganisations linked to this long conversion.

Keywords :

Industry, textile, globalisation, conversion, Vosges mountain, Lorraine, Alsace, France

Prononcée lors d'un entretien par une spécialiste du textile dans les Pays en Voie de Développement, la question utilisée comme titre résume la vision générale d'une branche et d'un bassin industriels en crise depuis plus de 40 ans et dont les ultimes survivants seraient promis à une disparition rapide avec la fin, au $1^{\mathrm{er}}$ janvier 2005, des accords multifibres qui limitaient les importations. L'idée que cette branche industrielle, après avoir été un pilier économique du Massif vosgien durant une centaine d'années, est en voie de disparition, semble donc acquise, malgré la réactivation partielle des quotas dès la mi-2005.

Ce déclin s'inscrit plus largement dans un contexte post-fordiste de mutations industrielles majeures, voire - pour certains - de disparition de l'industrie au sens classique du terme dans les pays développés. Maintes fois étudié, notamment dans le contexte de la mondialisation (Guillochon, 2003 ; Baudrand, Henry, 2006) et heureusement souvent nuancé (Battiau, 1998 ; Holz, Houssel, 2002; Daviet, 2005; Fache, 2006), ce déclin affecte particulièrement les branches industrielles anciennes, que l'on peut qualifier de traditionnelles tant elles ont conditionné le fonctionnement territorial de certains bassins ou vallées industrielles. C'est le cas des vallées vosgiennes, marquées par l'industrie textile, appellation 
commode $^{1}$ qui regroupera dans ce travail les classes 17 et $18.2^{2}$ de la Nomenclature des Activités Françaises (NAF), couvrant toutes les étapes de la chaîne de production et ses àcôtés : filature (17.1), tissage (17.2), ennoblissement textile (17.3), fabrication d'articles textiles (17.4), autres industries textiles (17.5), fabrication d'étoffes à mailles (17.6), fabrication d'articles à mailles (17.7), fabrication de vêtements en textile (18.2) - même si cette dernière ne répond pas à la définition précise du textile, elle lui est cependant fortement liée et «la complémentarité entre les deux branches est évidente »(Battiau, 1985).

L'aire d'étude demande également à être délimitée, car « Vosges » s'applique tant au département qu'au massif qui lui a donné son nom. L'industrie textile concerne en fait essentiellement la partie sud du massif et s'étend à la fois sur le versant oriental (alsacien), occidental (lorrain) et méridional (franc-comtois). Elle s'étend également pour des raisons historiques et techniques ${ }^{3}$, hors des limites du massif proprement dit et au-delà des communes classées en zone de montagne, de piémont ou bénéficiant du classement «Massif vosgien » du fait de leur contiguité au massif ou de leur adhésion au syndicat mixte du Parc Régional des Vosges du Nord (figure 1). C'est cette concentration textile débordant du massif, mais centrée sur la montagne, qui fait l'objet de ce travail ${ }^{4}$.

Le propos de cet article est de faire le point - dans une perspective géographique et donc plus spatiale qu'économique - sur la situation à la fin de 2006, après deux ans d'ouverture du marché et de réactivation partielle des quotas. Combien reste-t-il d'établissements textiles ? Quelles sont leurs spécialités ? Combien de personnes sont encore employées dans cette branche ? Quels sont les sites encore en production aujourd'hui ? Que sont devenus les sites abandonnés ?

Les sources de base utilisées pour répondre à ces questions sont tout d'abord les inventaires INSEE (fichier SIRENE) et ceux des CCI des départements concernés. Les listes proposées par ces organismes sont régulièrement mises à jour, mais comportent des incohérences. Dans ce cas, des vérifications ont été effectuées localement. Ce travail s'appuie également sur les données nationales consultables sur différents sites internet et en particulier celui du Ministère de l'Economie, des Finances et de l'Industrie (www.industrie.gouv.fr). Enfin, outre de nombreuses autres sources bibliographiques, cette étude utilise aussi un article fondamental qui présentait la situation - encore loin d'être catastrophique - de l'industrie textile des Vosges en 1979 (Thouvenin, 1979). Dans cet article étaient recensés - et très

\footnotetext{
${ }^{1}$ «Est textile le matériau qui se présente sous la forme de fibres séparables susceptibles d'être filées, c'est-àdire que ces fibres doivent être capables de former un ensemble dont la cohérence est due aux forces de friction qui existent entre ces éléments » (Battiau, 1985).

${ }^{2}$ Les catégories 18.1 (fabrication de vêtements en cuir) et 18.3 (industrie des fourrures), de toutes façons absentes de l'aire d'étude, sont exclues de ce travail.

${ }^{3}$ Le textile dit vosgien est en fait né à Mulhouse dès l'époque Moderne et s'est répandu dans le massif via les commerçants mulhousiens profitant d'une main d'œuvre libre l'hiver. La pénétration s'est faite par les vallées du versant alsacien (Thur, Fecht...), puis a franchi la crête vers les hautes vallées lorraines (Moselle, Moselotte, Meurthe...), avant de descendre ces vallées, notamment celle de la Moselle (en contrecoup de l'intégration de l'Alsace à l'Allemagne en 1871) jusqu'au-delà d'Épinal et donc bien au dehors du massif. De très nombreuses sources existent, retraçant cette épopée industrielle, en particulier les livres de G. Poull (Poull, 1982 ; Poull, 1997) ou de P. Durupt (Durupt, 1990).

${ }^{4} \mathrm{Ce}$ choix géographique pose un problème statistique car les données sont en général disponibles au niveau régional ou départemental, incluant donc des valeurs qui sont hors de l'aide d'étude et qu'il n'est pas toujours possible de différencier.
} 
Figure 1 : L'aire d'étude, un massif pour trois régions

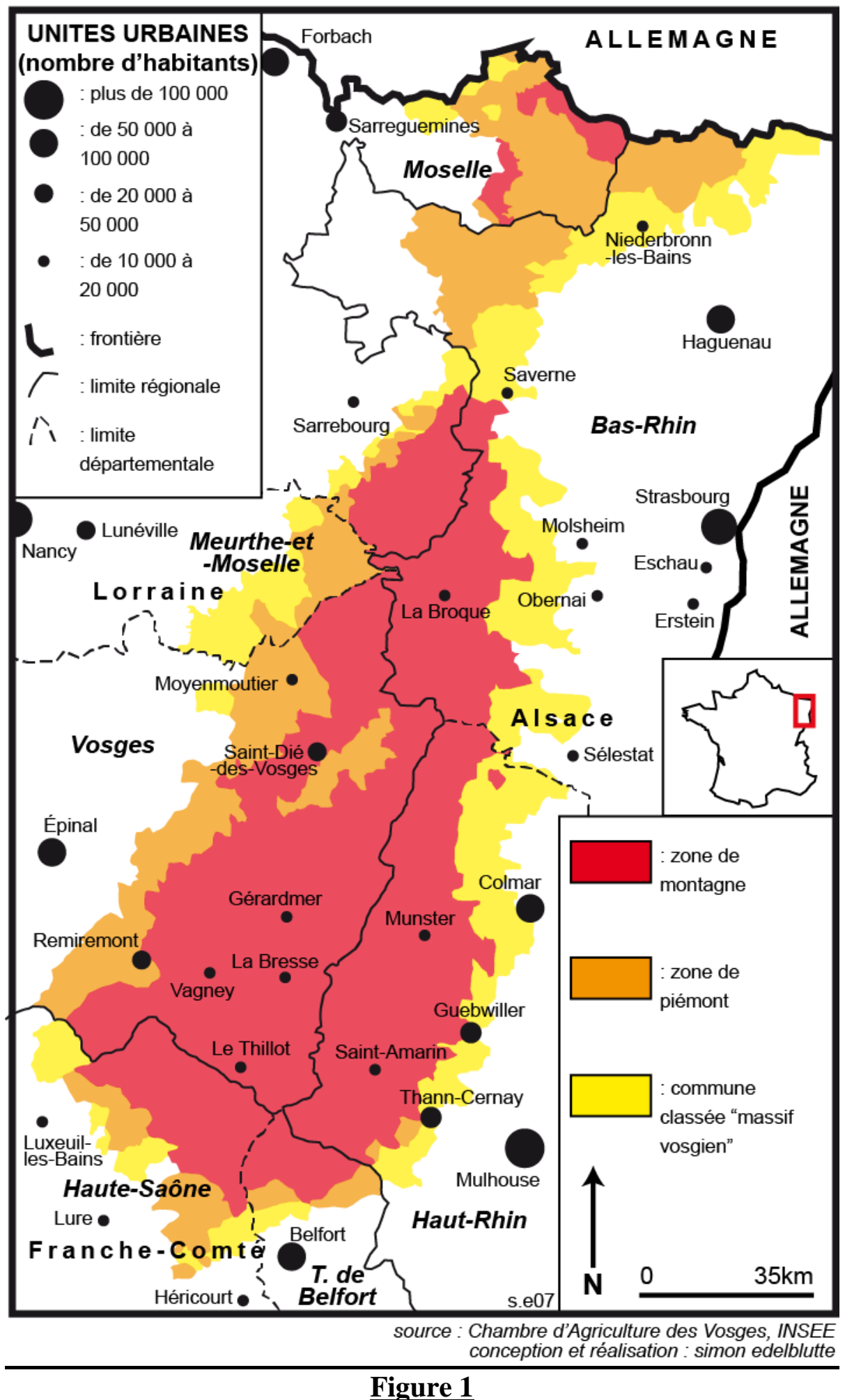

L'aire d'étude, un massif pour trois regions

The territory of the study: one mountain, three "régions"

finement cartographiés - tous les établissements textiles du bassin, avec leur type de production, leur effectif, leurs liens économiques... C'est donc un point de départ incontournable pour ce travail qui dressera tout d'abord un tableau global de la situation en la comparant à celle de 1979, avant de s'intéresser aux survivants de la débâcle - et plus 
particulièrement à ce qui leur a permis de survivre, voire de se développer - pour enfin et à plus grande échelle, étudier les différents sites et leur évolution.

\section{1 - Une filière affaiblie, mais toujours présente}

\section{1 - Un poids économique et spatial fort}

Au niveau national, le massif vosgien reste un bassin textile essentiel. En effet, en 2004 et dans le domaine des premières transformations (filature, tissage et ennoblissement ${ }^{5}$ ) la Lorraine, avec 8,6\% des entreprises de plus 20 salariés et 9,8\% des effectifs nationaux, et l'Alsace, avec 3\% et $7,5 \%$, tiennent respectivement les troisième et cinquième places nationales au niveau des effectifs, derrière Rhône-Alpes $\left(n^{\circ} 1\right)$, Nord-Pas-de-Calais $\left(n^{\circ} 2\right)$ et Midi-Pyrénées $\left(\mathrm{n}^{\circ} 4\right)$. La Lorraine assure ainsi plus de la moitié de la production nationale des filés et tissés, et ce, depuis plus de 10 ans (www.insee.fr). Elle emploie par ailleurs, en 2002, 8900 personnes dans le textile-habillement avec un indice de spécificité de $1,16^{6}$; cet indice monte à 2,37 pour la branche filature et tissage (Hublau, 2002).

Ces résultats appellent cependant de nombreuses nuances :

- tout en étant récents, ils datent d'avant la fin des quotas et leur réactivation partielle de 2005 ;

- ils concernent les régions entières et non le seul Massif vosgien. Toutefois, la figure 2 montre qu'en Lorraine comme en Alsace, l'essentiel des établissements textiles se trouve bien dans l'aire d'étude ;

- ils ne concernent qu'une partie - certes essentielle et emblématique - de l'industrie textile. Dans les autres domaines de l'industrie textile listés en introduction (17.4 à 17.7 et 18.2), la Lorraine est exclue des 5 régions de tête au niveau national, alors que l'Alsace n'apparaît qu'en $5^{\text {ème }}$ place de la fabrication d'articles textiles $(17.4)^{7}$, avec $6,4 \%$ des effectifs nationaux de la branche. De même, la Franche-Comté, pourtant première région de France en terme d'emplois industriels par rapport à la population active, n'est jamais présente dans ces palmarès textiles.

Malgré ces nuances, la branche textile est donc toujours très bien représentée dans le Massif vosgien d'autant plus que de grands groupes nationaux sont présents aux côtés de nombreuses PME. En effet, plusieurs sites du massif font partie des 10 premières entreprises nationales de leur catégorie. Ainsi, «Ganzoni » à Saint-Louis et «Tricotages des Vosges » à Vagney pour le travail de la maille (17.6 et 17.7); «Faurecia» à Marckolsheim et «Descamps » à Autmonzey, respectivement $\mathrm{n}^{\circ} 1$ et $\mathrm{n}^{\circ} 2$ pour les autres articles textiles $(17.4$ et $17.5)$; enfin « DMC (Dollfuss, Mieg et Compagnie) », groupe né à Mulhouse en 1746 et $\mathrm{n}^{\circ} 1$ du secteur filature, tissage et ennoblissement (17.1 à 17.3), possède 5 sites sur le versant alsacien du massif, regroupant plus d'un millier d'employés. L'essentiel des établissements les plus importants (entre 200 et 500 employés) est d'ailleurs contrôlé par des entreprises d'envergure nationale, comme «DMC » à Mulhouse ou Saint-Amarin, «Paul Hartmann SA » à Lièpvre ou Châtenois, "Tricotages des Vosges » à Vagney, "Linvosges » à Gérardmer ou encore «Bihr Frères Filature, Ficellerie et Corderie de l'Est » à Uriménil.

\footnotetext{
${ }^{5}$ NAF 17.1 à 17.3

${ }^{6}$ Cet indice calculé par l'INSEE signifie que le poids de l'emploi de ce secteur par rapport à l'emploi total est 1,16 fois plus important en Lorraine qu'en France.

${ }^{7}$ Cette catégorie correspond à la fabrication de produits finis non destinés à l'habillement (linge de maison, bâches, housses, tapis, moquette, ficellerie, corderie...).
} 
Figure 2 : Les établissements de plus de 10 salariés du secteur textile-habillement et leurs spécialités dans le Massif vosgien et ses abords (août 2006)

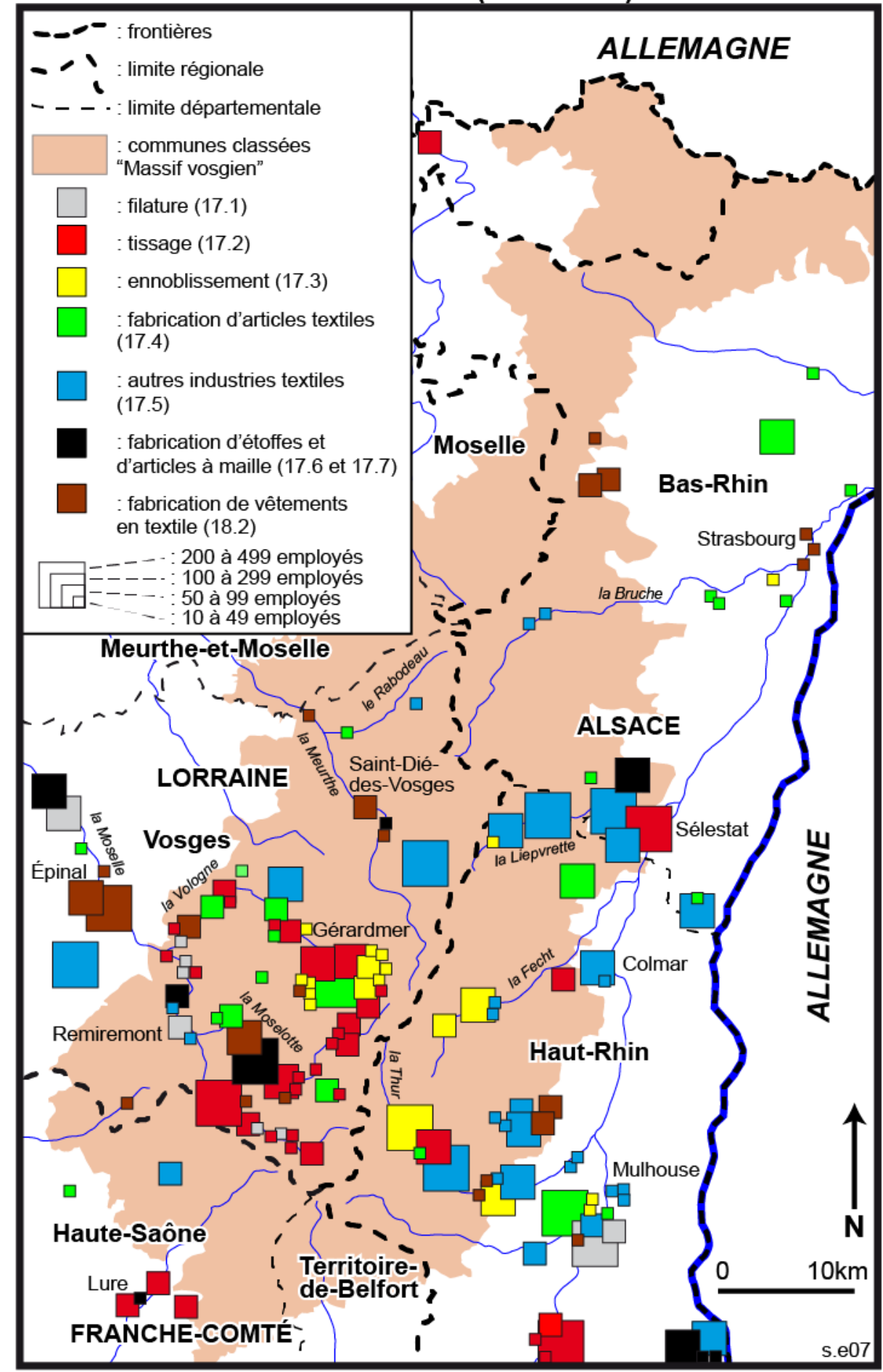

sources : fichiers consulaires des CCI 54, 57, 67, 68, 70, 88, 90 ; www.insee.fr conception et réalisation : simon edelblutte

\section{Figure 2}

Les établissements de plus de 10 salariés du secteur textile-habillement et leurs spécialités dans le Massif vosgien et ses abords

Plants with 10 employees or more and their specialities in the Vosges mountain and surroudings 
D'autres entreprises, d'envergure moindre, possèdent plusieurs établissements dans le massif, souvent dans la même commune ou tout au moins dans une même vallée, comme «Paul Hartmann SA » dans la vallée de la Liepvrette, «Tissage Mouline Thillot (TMT) » au Thillot, «Tissus Gisèle » à La Bresse, «Société de Tissage de Gérardmer Garnier-Thiébaut (STG) » à Gérardmer, « Filature et Tissage de Saulxures-sur-Moselotte » dans la commune du même nom...

À plus grande échelle et comme le montre la figure 2, la concentration est encore plus sensible. L'essentiel des établissements textiles est situé dans la partie sud du massif - la plus élevée - ou à ses abords immédiats. La zone d'emploi de Remiremont-Gérardmer compte par

exemple, en 2004, 48 établissements textiles, soit un petit peu moins de $30 \%$ des établissements de la zone pour un tiers des emplois industriels dans les établissements de plus de 10 salariés. Ces 3218 emplois représentent aussi un peu plus d'un tiers des emplois textiles lorrains. Les concentrations sont particulièrement visibles dans les grandes vallées descendant de la ligne de crête vosgienne. Du côté alsacien, la Lièpvrette, la Fecht et surtout la Thur ; du côté lorrain, la Moselle, la Moselotte et surtout la Vologne, alors que la Meurthe est beaucoup moins concernée. Hors du massif, le pôle mulhousien, lieu historique de la naissance du textile vosgien, émerge encore largement alors que d'autres villes comme Épinal ou Colmar, Lure ou Sélestat ne présentent plus que quelques sites. Enfin quelques établissements plus isolés peuvent être repérés le long du Rhin (Strasbourg, Marckolsheim, Saint-Louis) ou le long de la Moselle à l'aval d'Épinal (Nomexy), ou encore dans la partie nord de l'Alsace (Haguenau, Saverne).

Ce bilan encore honorable ne peut cependant cacher les très importantes pertes de la branche, tant dans un passé récent que durant les années 2005/2006.

\section{2 - Un déclin incontestable}

Les 8900 emplois lorrains de la branche évoqués en 2002 étaient encore 10000 en 1999, 17000 en 1990 et 67000 en 1955 (Hublau, 2002) ...

L'histoire et les causes du déclin déjà ancien du textile, avec en particulier la perte des débouches coloniaux après 1960, est bien connue et ne sera pas reprise ici. La figure 3, réalisée d'après la carte de M. Thouvenin publiée en 1979, montre non seulement les sites actifs en 1979 dans les domaines du tissage, de la filature et de l'ennoblissement, mais aussi les très nombreux sites fermés entre les années 1950 et 1979, le tout dans un impressionnant foisonnement.

Les établissements situés hors des vallées principales sont déjà presque tous fermés, mais surtout, la comparaison avec la figure 2, même si elle est difficile car la figure 3 ne comporte pas d'indictions d'effectifs, est néanmoins saisissante. Elle l'est d'ailleurs d'autant plus que la figure 3 ne concerne que les établissements de la branche filature, textile et ennoblissement et que la figure 2 y ajoute les autres catégories évoquées en introduction. Dans les trois régions, certaines vallées, comme celle du Rabodeau, de la Meurthe, de la Doller ou de la Bruche ont été presque totalement vidées de leurs usines textiles. 
Figure 3 : L'état des branches filature, tissage et ennoblissement dans le Massif vosgien en 1979

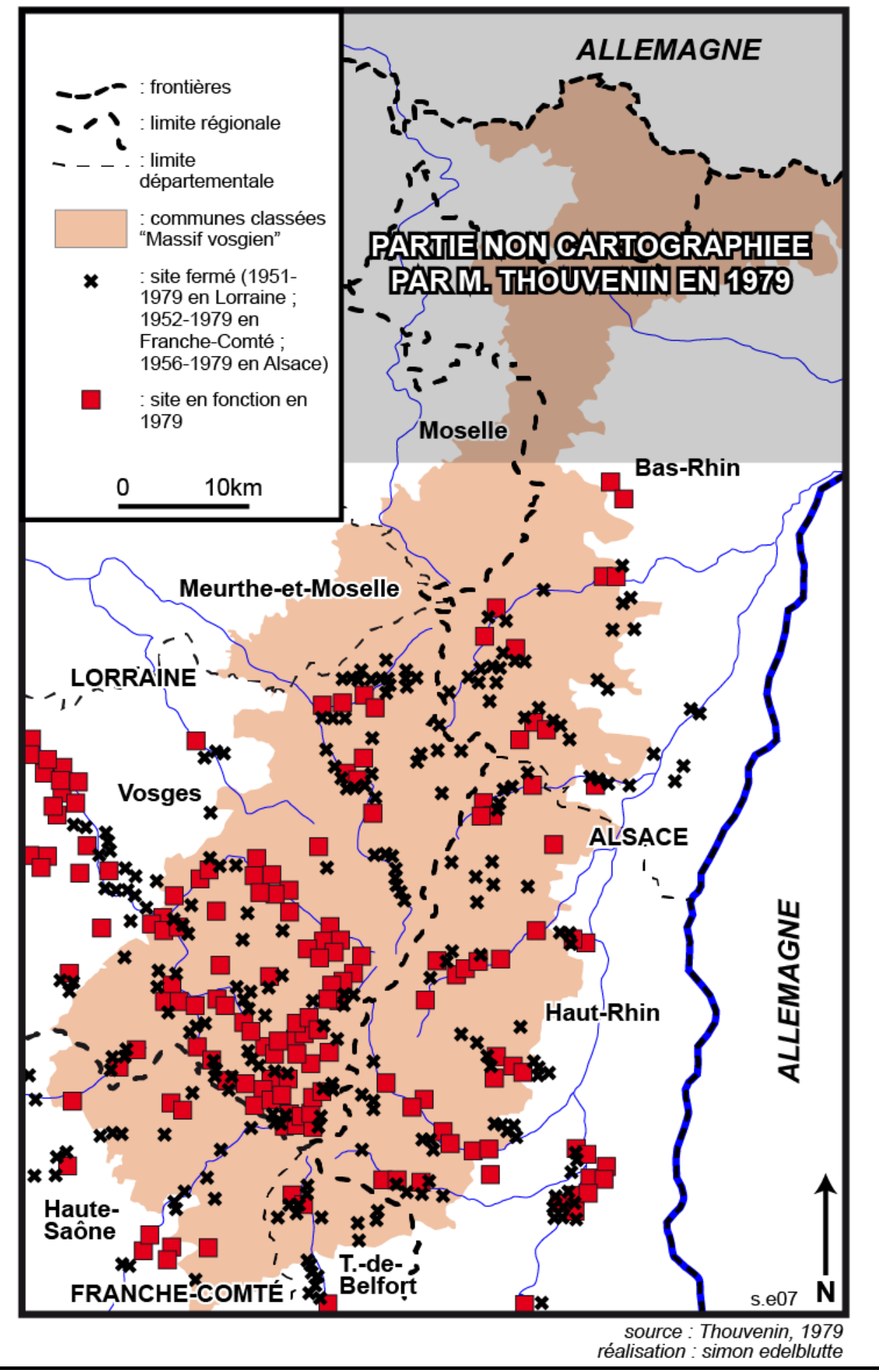

Figure 3

L'état des branches tissage, filature et ennoblissement dans le Massif vosgien en 1979

The state of the spinning, weaving and bleaching branches in Vosges mountain (1979)

La comparaison des deux figures permet aussi de repérer - notamment du côté alsacien - de nouvelles implantations à l'extérieur du massif (Sélestat), voire vers le Rhin 
(Marckolsheim, Saint-Louis), en prise directe avec les axes autoroutiers, délaissant ainsi les étroites vallées montagnardes à l'accès difficile.

Plus récemment, la fin des quotas limitant les importations a provoqué, et ce malgré leur réactivation partielle jusque fin 2007, un regain de la crise. On estime en mars 2005 les emplois de la branche (en Lorraine seulement) à 7000 contre 8900 en 2002... Plus encore, une comparaison entre la situation entre juin 2004 et janvier 2007 révèle une accélération du rythme des fermetures. Ce sont en effet 14 sites qui ont fermé, alors que parallèlement, de nombreux sites réduisaient leur activité. Enfin, le suivi régulier de l'actualité économique du massif au moment de la rédaction de ce travail révèle, plusieurs fois par mois, difficultés et fermetures, comme l'illustre l'encadré ci-dessous.

«Le textile vosgien continue de perdre des emplois. Depuis plusieurs années, la concurrence des produits en provenance des pays asiatiques a été néfaste pour des entreprises textiles. Ces effets se font sentir dans les Vosges : l'entreprise Eugène Georges au Val d'Ajol est en liquidation et la SA Matthieu et Fils à Deycimont est contrainte de licencier une partie de son personnel ».

La Tribune, 26 septembre 2006

«Vosges : Febvay Création en redressement judiciaire. La société implantée à Vagney est spécialisée dans la fabrication de vêtements pour les professionnels principalement l'hôtellerie-restauration. L'entreprise qui emploie 99 salariés a subi une baisse de commandes depuis le début de l'année. Elle est placée en redressement pour une durée de 6 mois ».

La Tribune, 17 octobre 2006

"Tenthorey va fermer son site de Pouxeux. 59 emplois vont être supprimés et l'activité textile sera recentrée sur l'usine d'Eloyes ».

La Tribune, 27 mars 2007

Dans ce contexte, la survie des certains établissements et donc le maintien de cette branche économique dans le massif semble largement compromis et ce, à moyen terme, confirmant le titre de cet article! Pourtant, on l'a démontré, ne serait-ce qu'avec la figure 2 , le textile vosgien est toujours présent en 2007. Quelles sont les conditions de sa survie, voire de son développement?

\section{2 - Faire face au déclin}

Face aux difficultés évidentes et incontestables, la survie passe par deux stratégies, parfois complémentaires :

- la spécialisation et l'innovation, notamment dans des types de production rares et/ou à haute valeur ajoutée ;

- le regroupement : classique par la concentration économique ou, plus innovant, par la mise en place d'un pôle de compétitivité autour du travail des fibres, regroupant filières bois et textile.

\section{1 - Innovation et spécialisation sont-elles salutaires?}

Les figures 2 et 4 montrent que, même si la «filature de l'industrie cotonnière (17.1A) » a pratiquement disparu, les fabrications traditionnelles sont encore bien présentes dans le massif . C'est le cas notamment du « tissage de l'industrie cotonnière (17.2A) » qui se maintient sur 33 sites, presque exclusivement sur le versant lorrain. Par ailleurs «l'ennoblissement textile (17.3Z)», spécialité soit alsacienne, soit gérômoise pour le blanchiment, est lui-aussi bien placé (19 sites). 
Cette répartition est classique dans le massif et est le fruit de l'antériorité de l'industrialisation textile alsacienne. Les sites alsaciens se sont en effet spécialisés plus tôt dans des branches plus innovantes et donc plus rentables, laissant le travail de base, filature et tissage aux usines lorraines, d'ailleurs souvent filiales des précédentes. La figure 4 confirme enfin la faible présence de la confection et de l'habillement, conformément à la tradition du massif.

Figure 4 : Les spécialtés textiles dans le Massif vosgien

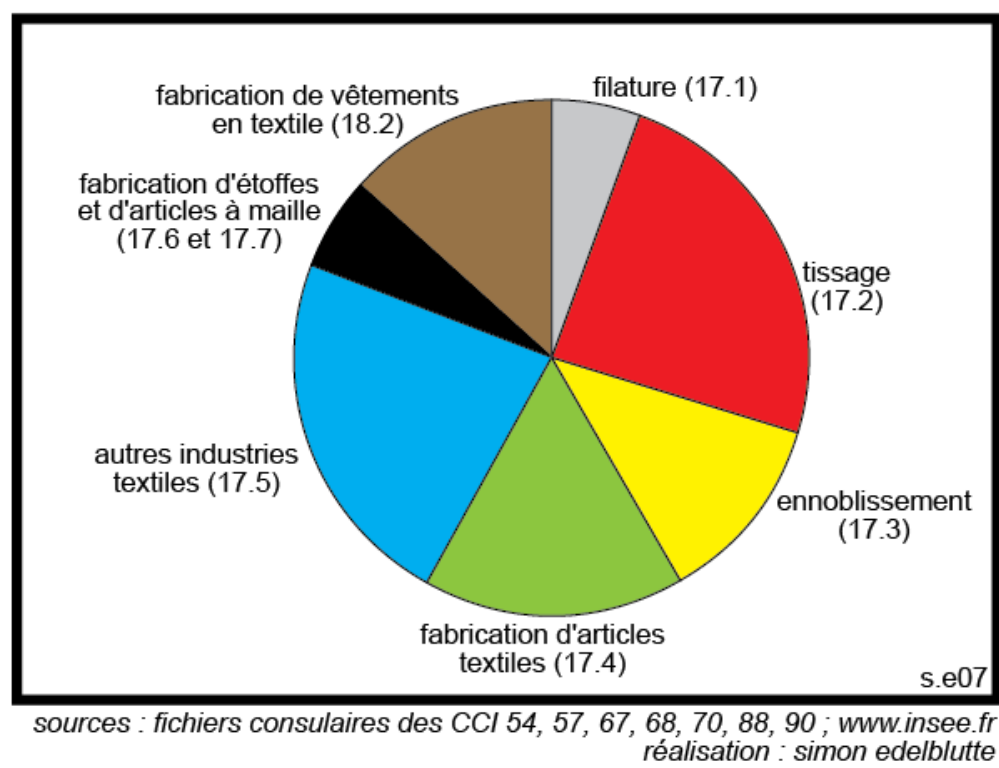

Figure 4

Les spécialités textiles dans le massif Vosgien

Textile specialities in the Vosges mountain

Parallèlement, une nouvelle spécialité, transversale car se retrouvant dans plusieurs catégories de la NAF - voire sortant du cadre du textile et de l'habillement et touchant, par exemple la plasturgie par la mise au point de matériaux composites intégrant des fibres textiles (Battiau, 2004-2005) - prend une importance croissante dans le massif, et, au-delà, dans la plupart des pays développés. Il s'agit des textiles techniques, désignant «des matériaux répondant à des exigences techniques et qualitatives élevées en matière de résistance mécanique, thermique et durabilité leur permettant de s'adapter à une fonction technique et à leur environnement (définition de H. Laurent et de G. Nemoz, de l'Institut Français du Textile et de l'Habillement). Le terme technique distinguant ces tissus, où les applications et les caractéristiques prévalent, des tissus plus esthétiques à vocation d'habillement et d'ameublement» (Moreau, 2005).

Ces produits textiles innovants, à forte valeur ajoutée et donc pour lesquels le coût de production n'est plus le critère déterminant, se démarquent des productions classiques aujourd'hui rentables dans les seuls pays émergeants ou en voie de développement. Ils sont particulièrement présents dans les catégories «Tissage d'autres textiles (17.2J)», «Fabrication d'autres articles confectionnés en textile (174.C)», « autres industries textiles (17.5) », avec en particulier les «non-tissés (17.5E) », mais ils sont présents aussi dans des branches plus classiques telles le «tissage de l'industrie cotonnière (17.2A)»; l'ensemble représente une quarantaine de sites. Parmi eux, peuvent être cités, à titre d'exemples : 
- «Interglass Technologies », entreprise allemande implantée à Malmerspach (68), dans la vallée de la Thur, fabriquant des tissus hybrides (carbone, fibre de verre et aramide) destinés au renfort d'autres matériaux ;

- «AK Filtration », à Rimbach-Zell (68), produisant des textiles filtrants pour les dépoussiéreurs industriels présents dans de très nombreuses industries ;

- «Decouvelaere », à Lépanges-sur-Vologne (88), produisant une fibre cellulosique naturellement extensible, tissu élastique à mémoire de forme...

La spécialisation accompagnée d'innovation peut également s'effectuer dans des créneaux plus classiques, en exploitant de niches ou en travaillant sur l'image et la commercialisation et en se spécialisant sur le haut de gamme.

Ainsi, «DMC », l'un des fleurons textile du massif, n'a dû sa survie et son développement dans les années 1990 que dans un recentrage sur le marché des loisirs créatifs en profitant du retour de la mode de la broderie, auparavant niche industrielle délaissée. Il emploie aujourd'hui plus de 1000 personnes dans cinq usines (après en avoir vendu une trentaine en 1996) et contrôle une chaîne de magasins (Loisirs et Création) où il commercialise ses produits avec succès.

Sur le versant lorrain, la saga de «Tricotages des Vosges », à Vagney, est fréquemment donnée en exemple. Cette ancienne usine Dim en difficulté est rachetée en 1994 par son ancien PDG qui compense ses coûts de production plus élevés en misant sur une production de chaussettes haut de gamme et en combinant une finition parfaite et une réactivité logistique que ses concurrents asiatiques ne peuvent atteindre. L'entreprise exporte $50 \%$ de sa production (www.economiematin.com, 2 octobre 2005) et a reçu en 2005 le trophée spécial du jury récompensant les dix entreprises françaises les plus dynamiques à l'international.

Enfin, l'e.commerce est également développé, à travers les sites de DMC pour les loisirs créatifs ou de Linvosges, entreprise gérômoise spécialisée dans la fabrication et la distribution de linge de maison, qui réalise aujourd'hui $15 \%$ de son chiffre d'affaires grâce à ses ventes en ligne.

Cependant, ce gros travail de spécialisation et d'innovation n'est non seulement pas à la portée de toutes les entreprises textiles du massif, mais, plus grave, il n'est pas non plus toujours garant de survie. Ainsi l'entreprise Decouvelaere, dont le patron a été le premier président du pôle fibres (qui sera évoqué dans la partie suivante) jusqu'à la mi-2006, est-elle depuis mai 2006 en redressement judicaire, son tissu révolutionnaire à mémoire de forme n'ayant pas encore rempli tous les espoirs commerciaux attendus. textile vosgien.

D'autres stratégies doivent donc être mise en œuvre pour préserver et développer le

\section{2 - S'unir pour survivre}

Les concentrations économiques opérées lors des crises textiles précédentes et poussées par les pouvoirs publics qui souhaitaient ainsi mettre en place des grands groupes que l'on pensait alors seuls susceptibles de survivre à la mise en place du Marché Commun (Battiau, 1985), ont montré leurs limites, avec notamment l'effondrement du géant BoussacSaint-Frères en 1981. Néanmoins, le regroupement plus réfléchi de sites textiles 
complémentaires et cohérents peut, en créant une dynamique et des économies d'échelles, préserver certains sites de la fermeture. Ainsi, 4 entreprises du massif, Le Tissage de la Mouline au Thillot (88), les Filatures et Tissages de Saulxures-sur-Moselotte (88), les Tissus Gisèle à La Bresse (88) et Le Tissage de la Côte à Lure (70), se sont rassemblées en 2006 au sein du groupe Incopar, pour "créer un groupe textile de taille européenne, susceptible de résister face aux importations liées à la mondialisation des marchés et d'être plus compétitif et réactif aux demandes des acheteurs textiles » (www.usinenouvelle.com, 22 juin 2006).

Plus que les regroupements, la clé de la survie semble être la mise en réseau, pratique déjà ancienne (le Syndicat Textile de l'Est existe depuis le $1^{\text {er }}$ mai 1890) mais aujourd'hui largement diversifiée. Ainsi a été créée Lorhatex (Lorraine Habillement Textile), fédération qui regroupe les industriels et partenaires de la filière textile en Lorraine afin de promouvoir le savoir-faire des entreprises de l'industrie textile et de mettre en oeuvre des actions collectives dans des domaines variés comme la veille technologique, la mise au point d'une stratégie commune, le travail sur la qualité...

Mais, dans ce domaine, la grande affaire actuelle est la labellisation du pôle de compétitivité «fibres naturelles Grand Est ${ }^{8}$, suite aux décisions du Comité Interministériel d'Aménagement du Territoire »(CIADT) en 2004 mettant sur pied des pôles se définissant par «la combinaison d'entreprises, de laboratoires de recherche et de centres de transferts, engagés sur un territoire donné dans une démarche partenariale d'innovation » (www.crlorraine.fr, 2005). Aux côtés de deux pôles plus purement textiles et tournés vers les textiles techniques et innovants en Rhône-Alpes (Techtera) et dans le Nord-Pas-de-Calais (UP-Tex), le pôle fibres, centré sur l'industrie des fibres cellulosiques, regroupe industries textiles et habillement (NAF 17 et 18), du bois (NAF 20 et 36) et du papier-carton (NAF 21), deux autres grandes spécialités du massif et plus largement de la Lorraine et de l'Alsace. Le tout représente, pour les deux régions dans leur ensemble, 1200 entreprises pour environ 50000 personnes.

Les objectifs affichés de conception de matériaux fibreux innovants (www.polefibres.uhp-nancy.fr) ont, évidemment et entre autres, un fort enjeu spatial pour un massif très forestier et de tradition textile. Certes, toutes ces industries ne sont pas dans le massif défini en introduction : l'industrie du meuble est ainsi essentiellement implantée dans l'ouest du département des Vosges (donc hors du massif) ; les papeteries, très présentes autour d'Épinal, le sont tout autant autour de Nancy et de Strasbourg. Cependant, l'axe fort du pôle fibres, Nancy-Épinal-Mulhouse, est bien centré sur le massif. Aux côtés des laboratoires de recherches de l'Université Henri Poincaré (UHP) de Nancy et des grandes écoles mulhousiennes, notamment de l'ENSITM (École Nationale Supérieure des Industries Textiles de Mulhouse), le campus fibres d'Épinal, avec l'ENSTIB (École Nationale Supérieure des Technologies et Industries du Bois) composante de l'UHP, du CRIITbois (Centre Régional d'Innovation et de Transfert de Technologie) et du CETELOR (Centre d'Essai du Textile Lorrain) - deux centres de transfert de technologies - semble en devenir la véritable capitale. L'ouverture, en septembre 2006 et sur ce campus fibres spinalien, d'une antenne du CTP (Centre Technique du Papier) de Grenoble permet d'associer la recherche papetière à celles du bois et du textile et renforce la place de cette ville au sein du pôle de compétitivité.

Il est évidemment encore un peu tôt pour juger de l'impact du pôle fibres sur la filière textile... d'autant plus que certaines entreprises textiles (Alcee, Febvay Créations,

\footnotetext{
${ }^{8}$ Communément abrégé en «pôle fibres ».
} 
Decouvelaere...) citées comme références dans la plaquette de présentation du pôle fibre datant de 2005 (www.pole-fibres.uhp-nancy.fr) sont aujourd'hui en difficulté...

Déclin textile et réactions au déclin textile ont donc profondément transformé la vie économique du massif vosgien depuis les années 1960. Le territoire, dans ses paysages et dans son organisation spatiale, en a été lui aussi profondément marqué.

\section{3 - Un bouleversement territorial}

Les vallées vosgiennes, aux paysages rythmés par les toits à sheds et les cheminées en briques des usines textiles (Doyen, 1983), présentent aujourd'hui un visage plus varié qu'autrefois, lié à une reconversion longue et encore en cours qui affecte dans un premier temps et en urgence les sites industriels eux-mêmes, mais aussi, dans un second temps et dans un processus plus global et plus réfléchi de redéveloppement des territoires (Daviet, 2006), les communes entières et, au-delà, les vallées.

\section{1 - Des usines à reconvertir, à moderniser, à préserver}

La reconversion est d'abord envisagée comme un processus de substitution des activités. Elle est entamée, pour le textile vosgien, dès les années 1960 et les premiers chocs de la crise textile. Elle s'accélère ensuite dans les années 1980, notamment avec l'effondrement de l'empire Boussac et ne peut en réalité jamais être considérée comme totalement terminée, dans un mouvement permanent de recomposition économique et spatiale.

Dans le massif vosgien, cette première phase est d'autant plus sensible que la crise y a été plus brutale que dans d'autres bassins industriels voisins (basin ferrifère, bassin houiller) ou des acteurs d'envergure nationale, comme par exemple Charbonnages de France, avaient prévu et géré la reconversion par anticipation.

Les premières actions, sensibles dès les années 1960, ont donc été de faire venir à tout prix et rapidement dans les vallées des emplois industriels de substitution aux emplois perdus. Cela peut d'abord se faire dans la branche textile elle-même, en accueillant une production textile plus moderne. Ainsi, l'arrivée, subventionnée par des aides de l'Etat, de la Communauté Economique Européenne et des collectivités locales, de Montefibre à SaintNabord (88), dans la banlieue nord de Remiremont, constitue dans ce domaine, un exemple presque caricatural de ratage de cette stratégie d'urgence. Construite en 1966 pour produire des textiles synthétiques, sur une nouvelle zone industrielle hors tissu urbain et bien reliée aux axes de communication, cette grosse unité a employé plus de 1000 personnes et était alors présentée comme le relais innovant et salvateur du textile vosgien traditionnel. Malheureusement, dans un contexte conjoncturel et structurel peu favorable, elle ferme définitivement en 1982 après 4 ans de difficultés, n'ayant donc fonctionné normalement qu'une dizaine d'années. La friche, immense (25ha), est rachetée par DMC qui n'en réoccupe qu'une partie et cède le reste au coup par coup à des entrepreneurs divers, sans plan d'ensemble. L'ancien site Montefibre est devenu aujourd'hui une sorte de patchwork industrialo-commercial mal entretenu, où subsistent ça et là des «dents creuses », dont l'ancienne implantation DMC elle-même, fermée en 1999, sonnant ainsi le glas de la vocation textile initiale du site. 
Plus fréquemment, on attire des industries autres que textiles, qui récupèrent parfois les bâtiments des anciennes usines fermés. Ainsi, à La Bresse (88), Eurelectric, fabrique de câbles filiale de Pirelli, s'implante au centre de la ville en 1962, dans d'anciennes usines textiles, avant, dans les années 1980, de s'exurbaniser dans deux anciens sites textiles horstissu urbain (Edelblutte, 2003).

Cette stratégie de diversification industrielle, largement utilisée dans les Vosges, notamment vers la papeterie, est plus solide à long terme que la précédente, mais peut non seulement mener à de nouvelles impasses en cas de délocalisation de la branche en question (ce qui se produit vers la Roumaine pour Eurelectric à La Bresse en 2006 www.usinenouvelle.com, 9 octobre 2006), mais aussi à un risque d'une trop grande dépendance vis à vis d'une autre branche industrielle, comme celle du papier par exemple.

Parallèlement, les sites textiles encore viables contribuent à maintenir une identité industrielle forte dans les vallées vosgiennes. Les entreprises innovantes citées dans la partie précédente sont, en effet et dans la grande majorité des cas, installées dans des bâtiments aux toits à sheds du début du siècle, entretenus et ainsi pérennisés.

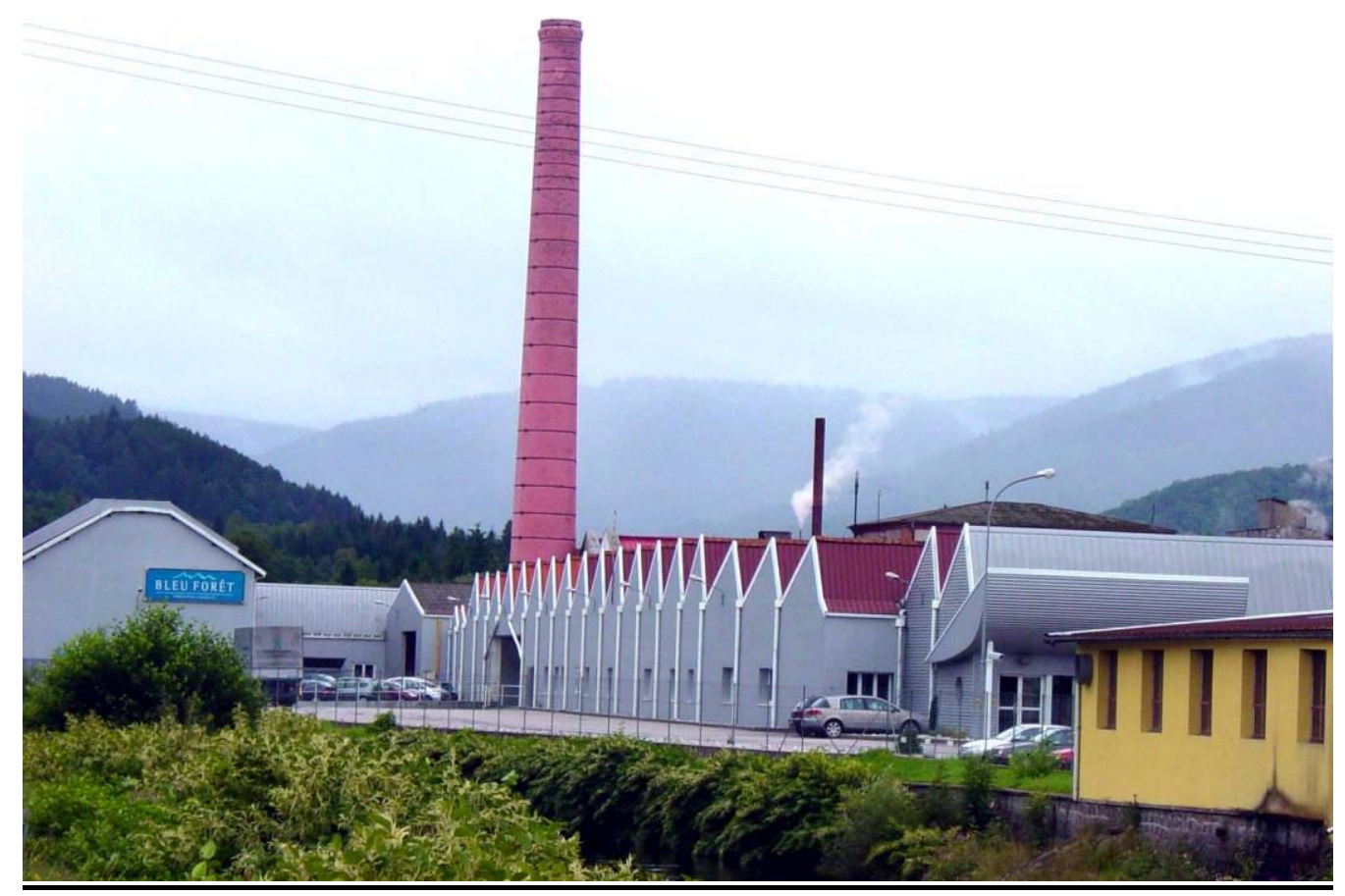

Photo 1 (Edelblutte, 2004)

L'usine « Tricotages des Vosges » à Vagney, Vosges

"Tricotages des Vosges" factory in Vagney, Vosges

Le bâtiment principal de cette usine a été construit en 1910, sur un site où l'industrie textile est présente depuis 1830. À la fin des années 1990, en liaison avec le recentrage de la production vers les chaussettes haut de gamme, il a été réhabilité par un bardage métallique. Un auvent moderne a été ajouté et la cheminée, entretenue et repeinte, est conservée dans un souci patrimonial d'authenticité. Le site emploie, en août 2006, 250 personnes.

S'il y a construction d'une nouvelle usine textile (résultant d'un transfert ou d'une création), elle se fait en général à travers un hangar métallique standard implanté à l'extérieur $\mathrm{du}$ tissu urbain sur une zone d'activités. Ce mouvement classique d'exurbanisation industrielle est particulièrement visible dans les communes situées hors du massif proprement 
dit. Ainsi, dans une vallée déjà assez large et profitant de sa présence sur l'axe mosellan, colonne vertébrale de la région lorraine, la commune de Nomexy (figure 5), au nord d'Épinal, a vu deux de ses trois sites textiles centenaires et centraux fermés (en 1997 et en 2003) et son troisième mis en liquidation judiciaire en novembre 2006, tandis qu'ouvrait en 1999 et sur une zone d'activités périphérique directement raccordée à la voie rapide Nancy-Épinal, l'usine Innothera (142 emplois), spécialisée dans les textiles médicaux (Noël, 2006).

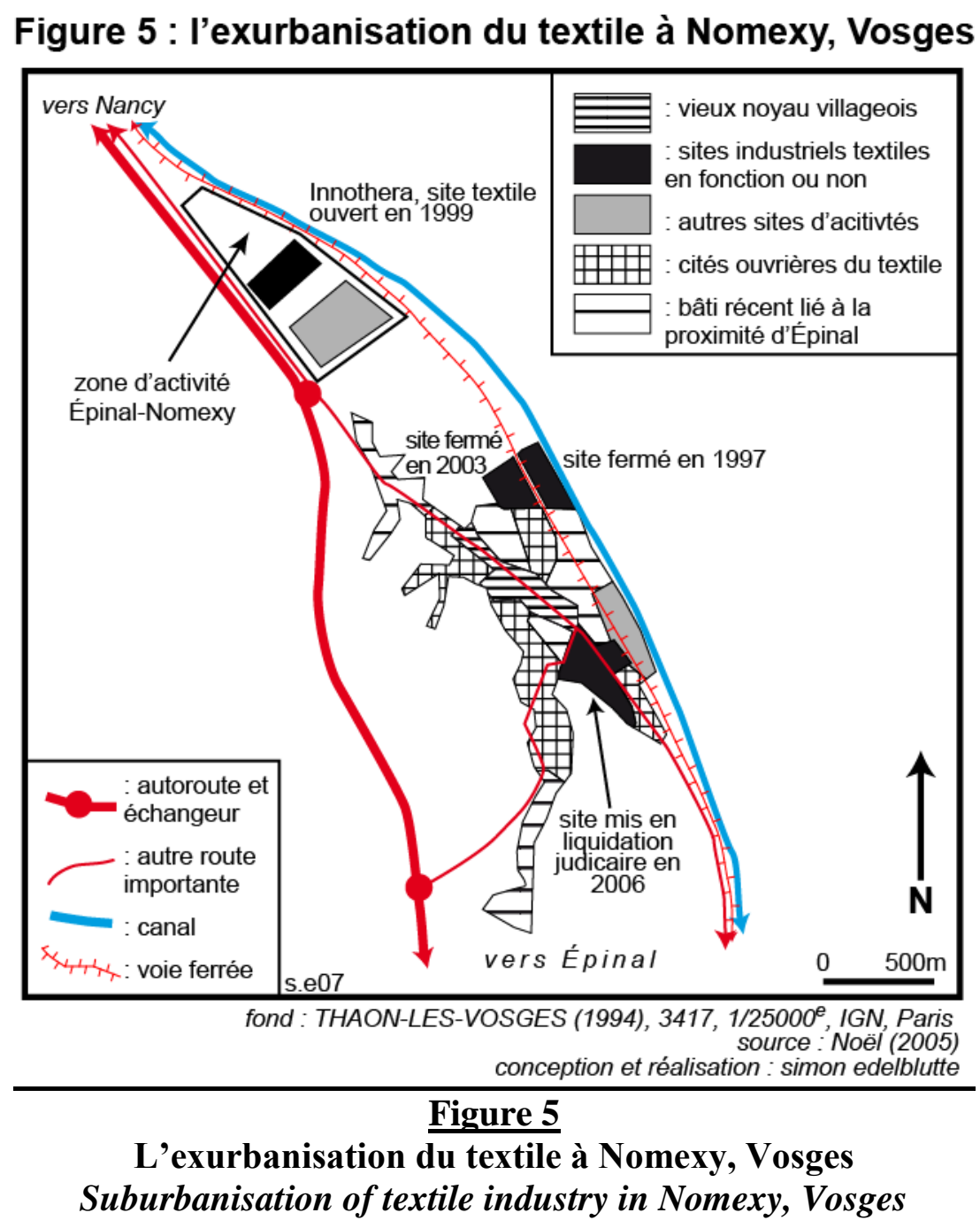

Enfin, certains sites abandonnés sont revitalisés dans une valorisation patrimoniale, participant ainsi au redéveloppement des territoires (cf. 3.2) par la préservation explicite de la mémoire textile du massif ; une identité nouvelle ne pouvant se forger sans la reconnaissance de ce qui a existé auparavant (Daviet, 2006).

Ainsi le Parc Naturel Régional des Ballons des Vosges (PNRBV) a-t-il coordonné un inventaire du patrimoine architectural lié à l'industrie textile, identifiant ainsi 320 sites (PNRBV, 2005). Par ailleurs, le PNRBV met en œuvre, depuis une dizaine d'années, de nombreuses actions visant à la fois à la connaissance et au développement de l'intérêt pour ce patrimoine, comme par exemple des randonnées cyclistes incluant la découverte, non seulement des sites anciens et actuels du textile, mais aussi des transformations paysagères, architecturales et urbaines que l'aventure textile a produit au cours des siècles précédents.

Le massif abrite en outre 4 musées textiles installés dans d'anciennes usines réhabilitées, à Val-et-Châtillon (54), Ventron (88), Sainte-Marie-aux-Mines et Wesserling (68). Ce dernier, 
le plus abouti, combine un musée classique à un parc industriel (en cours de réalisation) retraçant, dans un environnement de jardins fort prisé, l'histoire industrielle de la manufacture du temps des Lumières en 1762 à l'usine moderne fermée en 2003. Cet ensemble culturel est complété par une revitalisation économique (implantation de PME, de commerces) et résidentielle du site (Fluck, 2006).

Ce volet patrimonial de la reconversion montre aussi que la mutation rapide du textile vosgien a affecté non seulement les sites industriels eux-mêmes, mais aussi les villes et les vallées dans leur ensemble. La reconversion entre donc, depuis la fin des années 1980, dans une deuxième phase équivalant alors plus à un redéveloppement des territoires plutôt qu'à une simple substitution des activités.

\section{2 - Un modèle d'organisation spatiale fondamentalement remis en cause}

\section{Figure 6 : géosystème industriel, ville-usine, vallée industrielle}

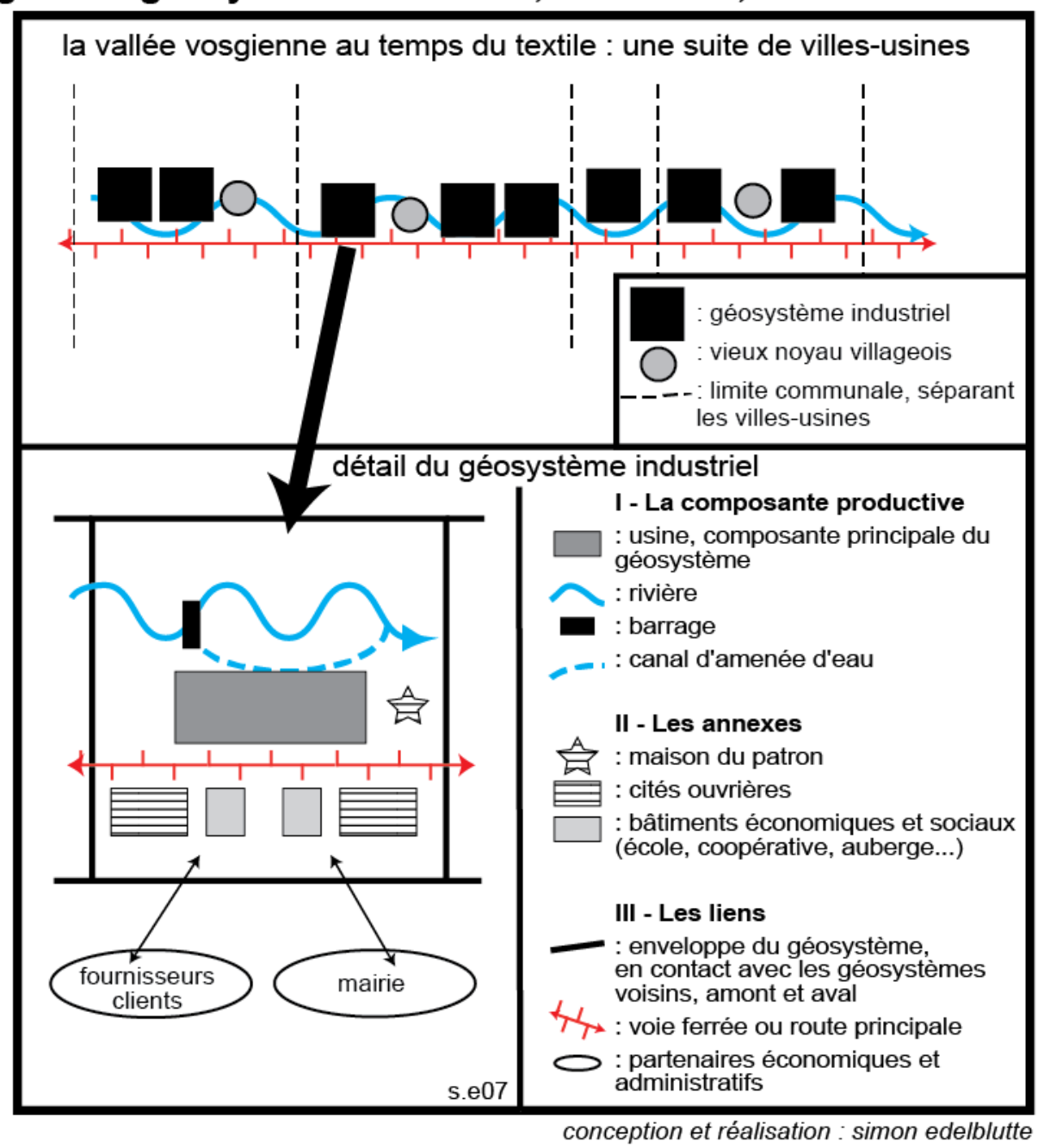

Figure 6

Géosystème industriel, ville-usine, vallée industrielle Industrial geosystem, company-town, industrial valley 
L'organisation traditionnelle de la vallée industrielle vosgienne était celle d'une suite de villes-usines ${ }^{9}$, nées sur un ou plusieurs géosystèmes ${ }^{10}$ industriels. Ces villes-usines sont, avec la croissance démographique et spatiale, entrées en continuité urbaine formant des conurbations (figure 6) linéaires, longs chapelets urbains, encore bien repérables dans les paysages actuels (photo 2).

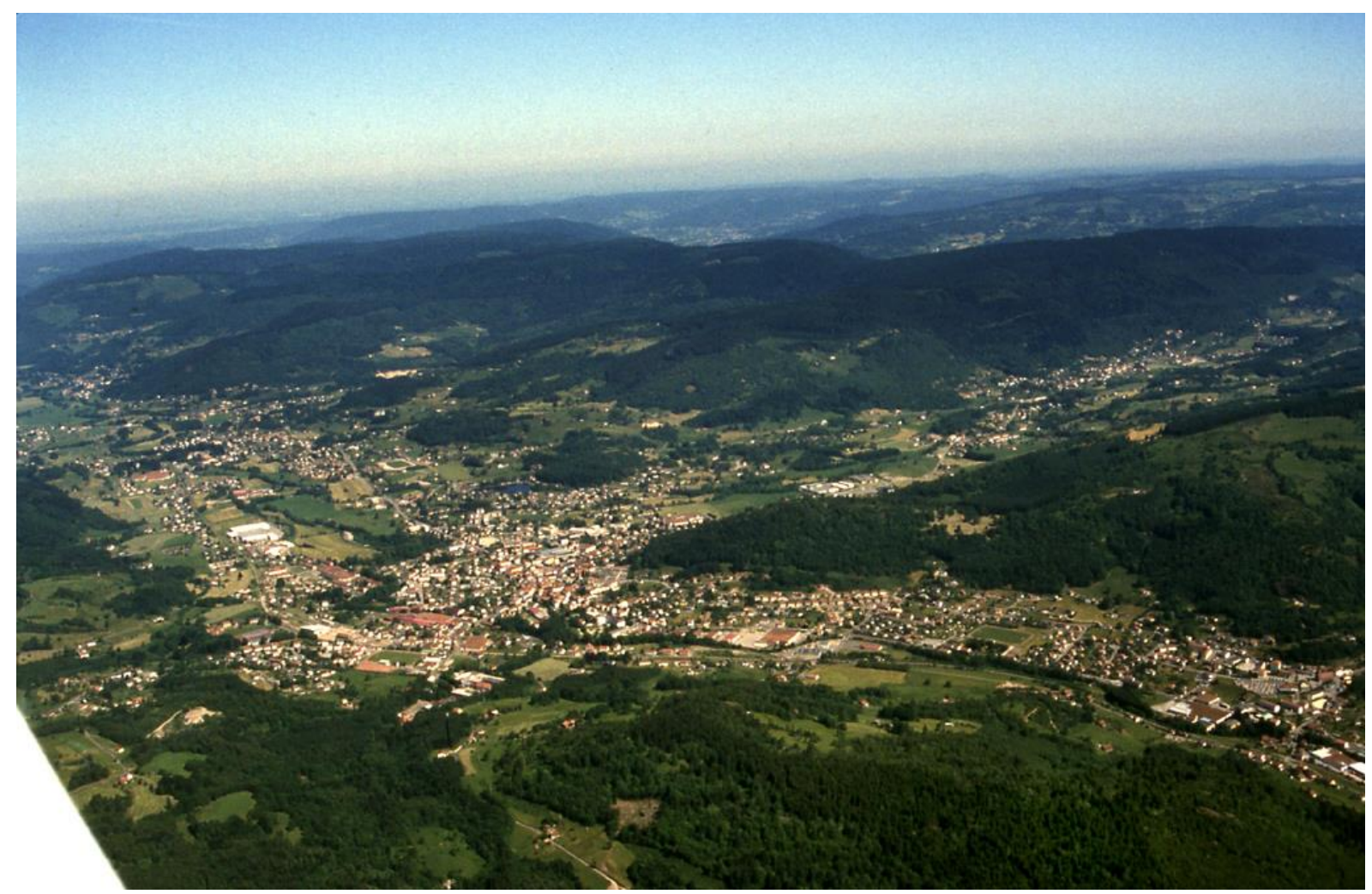

\section{Photo 2 (Humbert/CERPA, http://cerpa.univ-nancy2.fr, 1996) \\ Le chapelet urbain de la vallée de la Haute-Moselle autour du Thillot \\ The urban chain in Haute-Moselle Valley around Le Thillot}

Le cliché, centré sur la ville du Thillot (88) présente un chapelet urbain organisé linéairement dans la vallée de la Haute-Moselle. Les usines textiles, dont certaines sont encore en activité, sont entourées de cités ouvrières et de pavillons plus récents, le tout contribuant à la continuité urbaine.

Selon l'INSEE, ces agglomérations linéaires forment d'importantes unités urbaines, dépassant le plus souvent 10000 habitants. Le centre des ces unités urbaines est souvent difficile à définir car aucune des communes de ces unités urbaines ne domine vraiment les voisines. Par exemple, côté alsacien, l'unité urbaine de Saint-Amarin (Haut-Rhin, 10478 habitants $\left.{ }^{11}\right)$, dans la vallée de la Thur comprend 9 communes, réparties par l'INSEE en 4

\footnotetext{
${ }^{9}$ Une « ville-usine » est une ville presque totalement née de l'industrie paternaliste (à la différence d'une ville industrielle qui accueille des usines aux côtés d'un noyau urbain préexistant) et dont le cœur même était l'usinemère, entourée de cités ouvrières et de bâtiments économiques et sociaux (foyers, crèches, écoles, coopératives...) construits pour les ouvriers par l'usine (Edelblutte, 2006).

${ }^{10}$ Terme fréquemment utilisé en géographie physique pour désigner des systèmes produits par l'action conjuguée du relief, du climat et des sols, le géosystème est donc un espace cohérent aux éléments constitutifs interdépendants et aux liens étroits, le tout s'inscrivant dans le paysage. À ce titre, il peut être utilisé en géographie humaine pour désigner des ensembles homogènes et cohérents lisibles dans le paysage, tels les finages d'avant le déclin rural (Humbert, 1994), mais aussi les ensembles industriels nés autour des usines au XIXe siècle (Edelblutte, 2003) ou peut-être encore d'autres ensembles plus vastes, construits autour des agglomérations urbaines actuelles (Bertrand, 1999).

${ }^{11}$ Les chiffres INSEE du nombre d'habitants sont ceux du recensement de 1999.
} 
communes « centres » entre 1318 et 2440 habitants, et 5 communes de «banlieue » de moins de 1000 habitants. De même, côté lorrain, dans la haute vallée de la Moselotte, l'unité urbaine de La Bresse (Vosges, 13440 habitants), regroupe 5 communes, dont 3 « centres » et 2 «banlieues »; la commune-centre légèrement plus peuplée que les autres donne en général son nom à l'unité urbaine.

Ainsi, si la continuité urbaine, fondement de la définition de l'unité urbaine selon l'INSEE, est bien assurée et très visible (photo 2), il existait - et il existe toujours - dans ces ensembles industrialo-urbains étirés au fond des vallées, un manque évident de cohérence fonctionnelle. Chaque ensemble n'était donc que l'agrégat de multiples géosystèmes industriels, très cohérents à leur niveau, mais souvent rivaux à l'échelle de la vallée, voire de la commune, et cela même si la progression de la concentration dans le textile (constitution du groupe Boussac par exemple) avait permis l'élaboration de complémentarités entre les différents sites industriels d'une même vallée, voire à l'échelle du massif.

Cette organisation spatiale a évidemment été remise en cause assez brusquement avec la crise. La fermeture de l'usine signifie la disparition du cœur du géosystème, donc de l'élément fondamental qui faisait fonctionner tout le reste, entraînant non seulement l'apparition de friches industrielles, mais aussi une dégradation des habitats en cités ouvrières autrefois gérés et entretenus par l'usine. Même si l'usine ne ferme pas, voire continue à se développer, l'entreprise, pour des raisons évidentes d'économie, se désengage toujours du système paternaliste, fondement du géosystème industriel passé. Les bâtiments économiques et sociaux et les cités ouvrières sont vendus et personnalisés par leurs nouveau propriétaires, diversifiant un paysage autrefois assez monotone. Le pouvoir municipal, dans le passé inféodé, voire confondu avec la direction de l'usine, s'affirme peu à peu et prend en charge la reconversion au niveau local, en s'appuyant sur des aides régionales, nationales et européennes.

Ainsi, à l'échelle communale, le gros travail de réorganisation urbaine des ces anciens villes-usines textiles est ciblé sur ce qui a toujours manqué dans ces communes composées de la juxtaposition des petits géosystèmes industriels : la création ou le renforcement d'un véritable centre-ville, regroupant commerces et services et profitant souvent de la présence de friches centrales pour créer une place et/ou implanter quelques commerces (figure 7). C'est le cas à La Bresse, où deux friches textiles ont ainsi été réutilisées pour une piscine, un hôtelrestaurant et quelques résidences, renforçant un centre de plus en plus affirmé avec le développement de l'activité touristique (Edelblutte, 2003). Ce succès bressaud confirme la nécessaire diversification économique du massif vers les activités tertiaires, portées par le succès touristique du massif.

Parallèlement à cette réorganisation spatiale à l'échelle communale, l'intercommunalité se développe, à l'échelle des vallées. Le territoire des Communautés de Communes (CC), créées essentiellement durant les années 1990, correspond presque toujours dans le massif à une vallée ou une portion de vallée, tant du côté alsacien que lorrain. Les CC mettent donc peu à peu en place une cohérence fonctionnelle dans ces chapelets urbains nés de l'industrie textile (figure 7). Ainsi, la $\mathrm{CC}$ de la Haute Moselotte travaille à "l'harmonisation et la rationalisation du ramassage des ordures, à l'interconnexion des réseaux d'eau potable et au traitement des friches textiles » (www.ventron.fr). Néanmoins, la coopération, entre des communes autrefois économiquement rivales n'est pas toujours facile et certaines vallées, comme celle de la Haute Moselle autour du Thillot, sont seulement en cours de négociation pour la mise en place d'une telle structure à l'horizon 2007. C'est 
d'autant plus dommageable que "l'absence d'intercommunalité a souvent constitué un handicap » dans la reconversion (Daviet, 2006).

\section{Figure 7 : La nouvelle organisation spatiale des vallées vosgiennes}

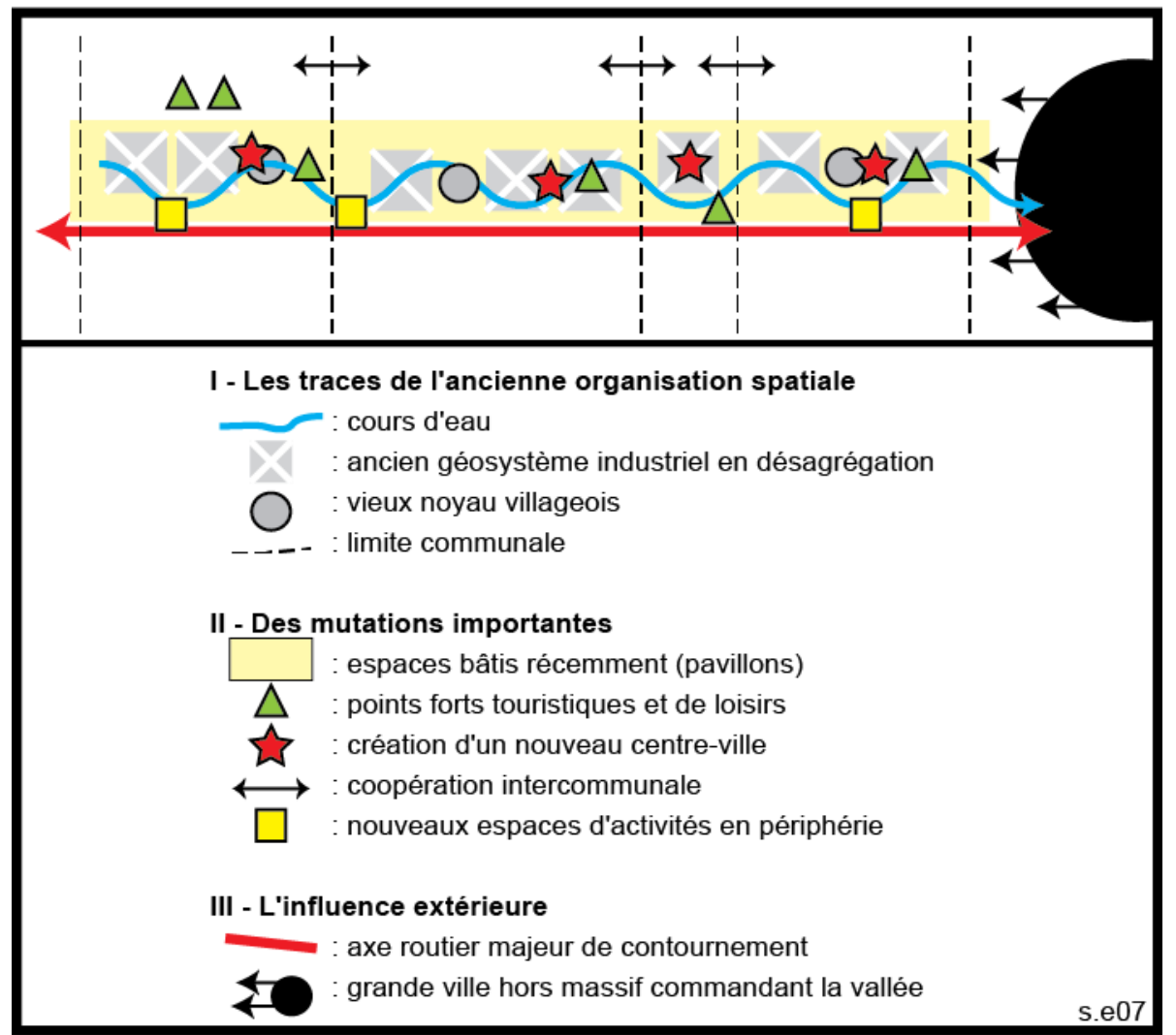

conception et réalisation : simon edelblutte

\section{Figure 7}

La nouvelle organisation spatiale des vallées vosgiennes The new spatial organisation of valleys in the Vosges mountain

\section{CONCLUSION}

La filière textile vosgienne, vieille de plus de 200 ans, a donc profondément marqué le territoire du massif et de ses abords, essentiellement dans sa partie sud. Elle continue aujourd'hui à fonctionner même si les menaces sur sa survie en tant que pôle industriel majeur du massif sont loin d'être écartées. Ainsi, aux côtés d'un patrimoine de plus en plus reconnu et mis en valeur et de réussites économiques portées par les innovations, la spécialisation et les coopérations diverses, son avenir reste toujours en question car ce nouveau paradigme industriel n'est pas encore intégré par toutes les entreprises textiles du massif (Jacquot, 1998).

Ces craintes sont cependant aujourd'hui de plus en plus mises à profit pour faire évoluer le massif dans sa globalité vers une diversification des activités toujours plus forte et vers une image toujours meilleure, notamment auprès des habitants eux-mêmes ; cette prise de conscience des atouts du massif par les habitants eux-mêmes est en effet l'une des clés de la revitalisation. Le massif vosgien, particulièrement du côté lorrain, a longtemps été perçu et avec quelques raisons - comme un bastion plutôt conservateur (dans un sens large, non politique) où la force d'inertie régnait en maître. Ainsi, l'époque des élus bressauds qui, au début les années 1980 et dans une commune qui est aujourd'hui (avec Gérardmer) le phare du 
tourisme vosgien, ne croyaient au salut que par la ré-industrialisation et pas par le développement touristique de la commune (Jacquemin, 1990), est heureusement aujourd'hui bien révolue.

La multiplication des initiatives patrimoniales, ou encore le développement - certes récent - des structures intercommunales, illustre ce changement de phase dans la vie d'un massif aujourd'hui largement désenclavé. Adossé à l'axe rhénan du côté alsacien, relié dès juin 2007 (Saint-Dié-de-Vosges, Épinal et Remiremont) à Paris par le TGV du côté lorrain, la montagne vosgienne peut donc largement profiter des dynamiques spatiales nationales et internationales qui se créent au cœur de l'Europe. La poursuite de la saga du textile vosgien, allant d'un foisonnement extraordinaire au déclin et aujourd'hui à la mise en œuvre de nouvelles stratégies, à la fois économiques et territoriales, est ainsi à l'image du renouveau du massif lui-même.

\section{BIBLIOGRAPHIE}

- Battiau Michel (1985), Le textile, vers une nouvelle donne mondiale, Dossier des images économiques du monde, Paris, Sedes, 233 p.

- Battiau Michel (1998), L'industrie. Définition et répartition mondiale, Paris, Sedes, 192 p.

- Battiau Michel (2004-2005), «Les impacts spatiaux de l'innovation dans les produits : l'exemple des textiles à usage technique dans le Nors-Pas-de-Calais », Cahiers Nantais, ${ }^{\circ}$ 62-63, p. 153-160.

- Baudrand Vincent, Henry Gérard-Marie (2006), Comprendre la mondialisation, Levallois-Perret, Studyrama, $180 \mathrm{p}$.

- Bertrand Vincent (1999), Les espaces rurbains en Lorraine : du paysage au système géographique - L'exemple du Lunévillois, Nancy, Thèse de Géographie, Nancy, Université Nancy2, 445 p.

- Daviet Sylvie (2005), Industrie, culture, territoire, Paris, L'Harmattan, 208 p.

- Daviet Sylvie (2006), «L'évolution du concept de reconversion : de la substitution des activités au redéveloppement des territoires ». In : Daumalin Xavier, Daviet Sylvie, Mioche Philippe - dir., Territoires européens du charbon, des origines aux reconversions, Aix-En-Provence, Publications de l'Université de Provence, p. 243-255.

- Doyen Jean-Pierre (1983), «Les villes-usines de la moyenne Moselle », Annales de la Société d'Émulation des Vosges, p. 52-71.

- Durupt Pierre (1990), Hommes et femmes du textile dans les Hautes-Vosges, Remiremont, Société d'Histoire Locale de Remiremont et de sa Région, 192 p.

- Edelblutte Simon (2003), «Géohistoire des paysages d'une vallée vosgienne ; l'exemple de la haute vallée de la Moselotte à La Bresse », Revue Géographique de l'Est, Tome XLIII, n³, p. 95-108.

- Edelblutte Simon (2006), «Renouvellement urbain et quartiers industriels anciens : l'exemple du quartier Rives de Meurthe / Meurthe-Canal dans l'agglomération de Nancy », Revue Géographique de l'Est, Tome XLVI, n³-4, p. 82-94.

- Fache Jacques (2006), Les mutations industrielles, Paris, Belin, 192 p.

- Fluck Pierre (2006), Wesserling (Alsace, France) : un patrimoine européen et un modèle de reconversion, XIII International Congress of The Industrial Committee for the Conservation of Industrial Heritage (TICCIH), www.ticcihcongress2006.net/paper/Paper\%204/Fluk.pdf

- Guillochon Bernard (2003), La mondialisation, une seule planète, des projets divergents, Paris, Larousse, $128 \mathrm{p}$.

- Holz Jean-Marc, Houssel Jean-Pierre (2002), L'industrie dans la nouvelle économie mondiale, PUF, Paris, $450 \mathrm{p}$.

- Hublau Isabelle (2002), «L’industrie textile résiste, l'habillement s’affaiblit », Économie lorraine, $\mathrm{n}^{\circ} 217$, p. $15-22$.

- Humbert André (1994), « Géographie historique ou la dérive des systèmes géographiques », Hérodote, $\mathrm{n}^{\circ} 74 / 75$, p. 95-111.

- Jacquemin Olivier (1990), La Bresse, commune touristique - Acteurs et enjeux, Mémoire de maîtrise, Nancy, Université Nancy2, 198 p.

- Jacquot Lionel (1998), Rationalisation du modèle de production et expérience du travail : l'industrie textile vosgienne à l'épreuve de la modernisation, Thèse de sociologie, Nancy, Université Nancy2, 424 p. 
- Moreau Arielle (2005), «Mondialisation dans le textile. Les atouts français : les textiles techniques, la conception et le marketing ». In : Collectif, L'industrie en France et la mondialisation, Paris, Ministère de l'Économie, des finances et de l'industrie, Statistiques et Études Industrielles (SESSI), 414 p. (Téléchargeable à ce lien : http://www.industrie.gouv.fr/biblioth/docu/mondial/mondial2005.htm)

- Noël Guillaume (2006), Nomexy, étude géo-historique et économique d'une ville industrielle textile des Vosges, Mémoire d'étape de MASTER de Géographie, Nancy, Université Nancy2, 84 p.

- Parc Naturel Régional des Ballons des Vosges (2005), Patrimoines industriels et techniques - Lieux de visite dans le PNRBV, Munster, PNRBV, 29 p.

- Poull Georges (1982), L'industrie textile vosgienne (1765-1981), Rupt-sur-Moselle, Édité par l'auteur, 474 p.

- Poull Georges (1997), Les fondateurs de l'industrie textile vosgienne, Metz, Éditions Serpenoise, 291 p.

- Thouvenin Monique (1979), « Aperçu sur l'industrie du coton et des fibres alliées dans le massif vosgien et sa périphérie », Revue Géographique de l'Est, Tome XIX, n³-4, p. 215-236.

\section{SITES INTERNET CONSULTÉS AU PRINTEMPS 2007}

- Conseil Régional de Lorraine, www.cr-lorraine.fr

- Économie Matin, www.economiematin.com

- Institut National de la Statistique et des Études Économiques (INSEE), www.insee.fr

- L'Usine Nouvelle, www.usinenouvelle.com

- Mairie de Ventron, www.ventron.fr

- Ministère de l'Économie, des finances et de l'industrie, Statistiques et Études Industrielles (SESSI),

www.industrie.gouv.fr

- Pôle de compétitivité fibres naturelles Grand Est, www.pole-fibres.uhp-nancy.fr 